\title{
Chemical Vapor Deposition Model of Polysilicon in a Trichlorosilane and Hydrogen System
}

\author{
G. del Coso, ${ }^{\mathrm{z}}$ C. del Cañizo, and A. Luque
}

Instituto de Energía Solar, E.T.S.I. Telecomunicación, Universidad Politécnica de Madrid, 28040, Madrid,

Spain

\begin{abstract}
The traditional polysilicon processes should be refined when addressing the low energy consumption requirement for the production of solar grade silicon. This paper addresses the fluid dynamic conditions required to deposit polysilicon in the traditional Siemens reactor. Analytical solutions for the deposition process are presented, providing information on maximizing the rate between the amount of polysilicon obtained and the energy consumed during the deposition process. The growth rate, deposition efficiency, and power-loss dependence on the gas velocity, the mixture of gas composition, the reactor pressure, and the surface temperature have been analyzed. The analytical solutions have been compared to experimental data and computational solutions presented in the literature. At atmospheric pressure, the molar fraction of hydrogen at the inlet should be adjusted to the range of $0.85-0.90$, the gas inlet temperature should be raised within the interval of 673 and $773 \mathrm{~K}$, and the gas velocity should reach the Reynolds number 800 . The resultant growth rate will be between 6 and $6.5 \mu \mathrm{m} \mathrm{min}{ }^{-1}$. Operation above atmospheric pressure is strongly recommended to achieve growth rates of $20 \mu \mathrm{m} \mathrm{min}{ }^{-1}$ at $6 \mathrm{~atm}$.

(C) 2008 The Electrochemical Society. [DOI: 10.1149/1.2902338] All rights reserved.
\end{abstract}

Manuscript submitted September 27, 2007; revised manuscript received March 5, 2008. Available electronically April 29, 2008

The strong photovoltaic (PV) market growth relies on crystalline silicon, using highly purified silicon as raw material, which is referred to as polysilicon. Traditionally, polysilicon for the solar industry has been obtained from the microelectronics industry, using off-spec material or the excess capacity of polysilicon producers. But the tremendous growth of the PV industry has produced a rapid change in the situation: while in 2000, PV only demanded $10 \%$ of the polysilicon, in 2005, PV demand surpassed that of the microelectronic industry, in the range of $15.000 \mathrm{t},{ }^{1}$ and the global demand exceeded the production capacity. Nevertheless, the silicon shortage that threatened PV industry is being overcome. This is resultant from initiatives taken by the polysilicon and the PV industry to keep on growing. Optimization of the purification process to address solar cell requirements must also be pursued in order to reduce the cost of the material as much as possible. For the moment, the consolidated route in the market to produce polysilicon is based on the synthesis and purification of silanes [monosilane (MS) or trichlorosilane (TCS)], and their subsequent reduction in a chemical vapor deposition (CVD) reactor to solid Si. Almost 77\% of the polysilicon produced worldwide is currently obtained from trichlorosilane in a CVD reactor known as a Siemens reactor. ${ }^{2}$

The Siemens reactor consists of a chamber where several highpurity silicon slim rods are heated by an electric current flowing through them, and polysilicon is deposited on the seed rods through the thermal decomposition of silanes in a hydrogen environment. The deposition features depend on the gas flux, and therefore, the fluid mechanics regime has to be analyzed profoundly to achieve the better flow conditions to grow polysilicon.

Analytical solutions for the deposition process of polysilicon are presented, based on the approach of splitting the second-order reaction rate into two systems of first-order reaction rate. The growth rate, deposition efficiency, and power-loss dependence on the gas velocity, the mixture of gas composition, the reactor pressure, and the surface temperature have been analyzed. The solutions presented have been compared to experimental data and computational solutions presented in the literature. Finally, it is discussed how to maximize the rate between the amount of polysilicon obtained and the energy consumed throughout the deposition process.

\section{Deposition Model}

There is a closed and adiabatic boundary between two neighboring rods inside the reactor as a result of the symmetries (i.e., neither mass nor heat can pass through). The shape of this boundary depends on the arrangement of the rods in the reactor (hexagonal,

${ }^{\text {z }}$ E-mail: gdelcoso@ies-def.upm.es circular, etc.). Then, a hollow cylinder has been considered to define the system because it is the most general boundary shape. The system geometry is shown in Fig. 1. The inner radius $r_{\mathrm{i}}$ defines a surface that corresponds to the polysilicon rod, and the outer radius $r_{\mathrm{o}}$ defines the closed and adiabatic boundary that corresponds to the equidistant space between neighboring rods, where neither mass nor heat can pass through. The height of the cylinder is $L$.

The transport phenomena and the surface reactions have to be taken into account to discuss the silicon growth rate. The concentrations of chemical species at the rod surface set, among other variables, the growth rate, and differ from those at the entrance to the reactor; therefore, they should be calculated by analyzing the transport phenomena. Some assumptions are made in order to aid in the solution of the transport and conservation equations: ${ }^{3,4}$ no axial diffusion of the properties, steady and laminar flow, ideal gas law, the gas transport properties are independent of the mass fraction of species $w_{i}$, the thermal diffusion and viscous energy dissipation are negligible, and the pressure is set as constant. A steady-state condition has been considered because, although deposition takes place

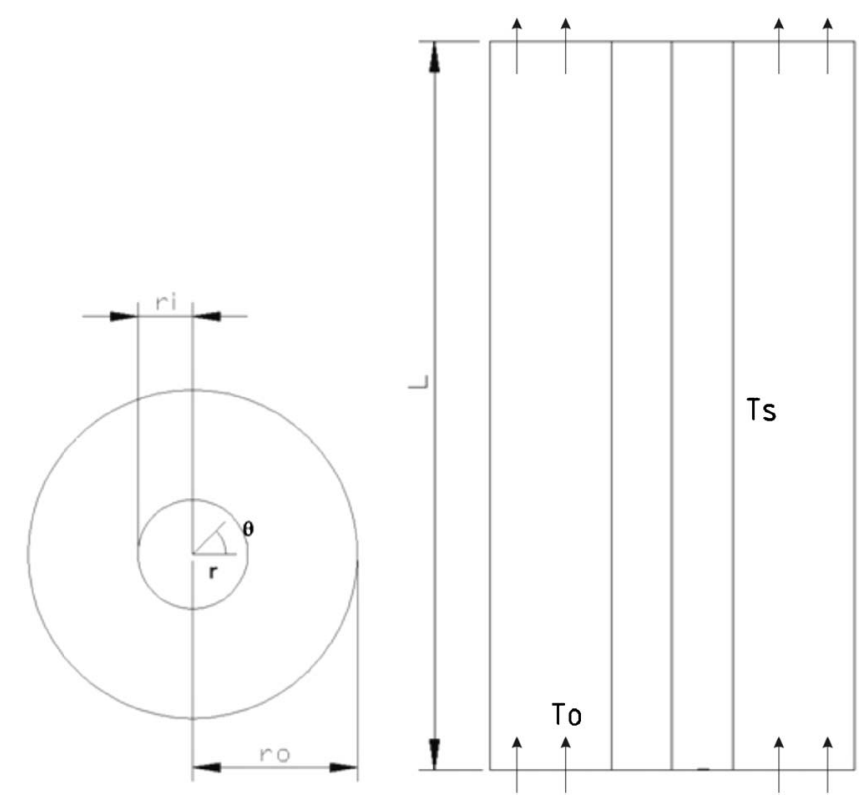

Figure 1. System geometry. Top and side views. $T_{0}=773 \mathrm{~K}, T_{\mathrm{s}}=1423 \mathrm{~K}$, $p=1 \mathrm{~atm}, L=1.5 \mathrm{~m}, r_{\mathrm{o}}=15 \mathrm{~cm}$, and $r_{\mathrm{i}}=5 \mathrm{~cm}$. 
and changes the geometry, the mass fraction and temperature profile are set faster. Cylindrical coordinates, centered in the base of the cylinder, are chosen to solve the equations. The properties of the gas mixture, which have to be known to solve the equations presented below, have been calculated following the approximations presented in the literature. ${ }^{5-8}$

Velocity profile.- The velocity profile of the gas mixture across the reactor is calculated by means of the mass conservation equation and the linear momentum conservation equation in axial direction

$$
\begin{gathered}
\frac{\partial}{\partial z}\left(\rho v_{z}\right)+\frac{1}{r} \frac{\partial}{\partial r}\left(r \rho v_{\mathrm{r}}\right)=0 \\
\rho\left(v_{\mathrm{r}} \frac{\partial v_{z}}{\partial r}+v_{z} \frac{\partial v_{z}}{\partial z}\right)=-\frac{\partial p}{\partial z}+\mu \frac{1}{r} \frac{\partial}{\partial r}\left(r \frac{\partial v_{z}}{\partial r}\right)+\frac{4}{3} \mu \frac{\partial^{2} v_{z}}{\partial z^{2}}-\rho g
\end{gathered}
$$

It should be noted that $p$ has not yet been set as a constant. The energy conservation equation and mass species conservation equation are also required to obtain the velocity profile because both determine the $\rho$ dependence on temperature and spatial coordinates. Despite that, the reasoning presented below is focused on Eq. 1 and 2 because it is based in the Heaton et al. ${ }^{9}$ analytical solution of these equations for incompressible fluids and their calculated hydrodynamic entry length. Some conclusions can be derived from this analysis even though, in our study, the fluid properties are not constant with temperature and the fluid is compressible (i.e., the density depends on the temperature and spatial coordinates): the lower the viscosity of the fluid the larger the entry length. The gas mixture has actually lower viscosity than incompressible fluids presented in the aforementioned study ${ }^{9}$ and, thus, a higher entry length. By setting the reactor length lower than the entry length, the boundary layer would be thin and not fully developed and, therefore, the radial component of the gas velocity could be ignored because it only exists within this thin layer.

The warming of the gas mixture also has to be taken into account: the gas closer to the rod increases its temperature, and consequently, its density decreases and its velocity increases. The increase in the velocity in that region makes the boundary layer even smaller where the radial component has to be considered. Thus, it can be stated that the radial velocity and the boundary layer can be ignored.

After ignoring $v_{\mathrm{r}}$, the equations can be solved separately. Equation 1 yields

$$
\frac{\partial}{\partial z}\left(\rho v_{z}\right)=0
$$

The boundary condition is $\left.v_{z}\right|_{z=0}=v_{0}$ and the solution to this equation, $\rho_{0}$ being the density of the gas at the entrance of the reactor, is

$$
v_{z}=v_{0} \frac{\rho_{0}}{\rho} \Leftrightarrow v_{z}(r, z)=v_{0} \frac{T(r, z)}{T_{0}}
$$

Note that the value of this solution at the rod surface is not zero because the assumption is that the boundary layer is small enough to be ignored. In the boundary layer, the velocity components go from a certain value at the border of the layer to zero on the surface, but taking the limit as the layer thickness as zero the value of the axial velocity at the surface is not zero.

The equation of linear momentum conservation, whose axial component is shown in Eq. 2, can be used to obtain the pressure field within the reactor, validating the assumption that the pressure is constant.

Heat transfer.- Some aspects, such as the maximum temperature permitted to avoid the gas phase reactions or the optimization between power loss and growth rate in the reactor, demand that the temperature distribution be obtained in the mixture of gas. Thus, the energy conservation equation is used to obtain this temperature distribution

$$
\rho C_{\mathrm{p}} v_{z} \frac{\partial T}{\partial z}=\frac{1}{r} \frac{\partial}{\partial r}\left(\kappa r \frac{\partial T}{\partial r}\right)
$$

The boundary conditions for $T$ are as follows: (i) the temperature of the gas on the surface of the rod is $T_{\mathrm{s}}$, (ii) adiabatic condition in $r_{\mathrm{o}}$, and (iii) constant and known temperature distribution at the reactor entrance $T_{0}$. The solution of Eq. 5, which is derived in Appendix A, is

$$
\Delta T(r, z)=\Delta T_{0} \sum_{n=0}^{\infty} A_{n} \exp \left(-\frac{\theta_{n}^{2} \kappa z}{\rho_{0} v_{0} C_{\mathrm{p}}}\right) \chi_{n}(r)
$$

where $\Delta T(r, z)=T(r, z)-T_{\mathrm{s}}$. The convection is the heat transfer mechanism that takes place in a fluid because of the heat conduction and the energy transport as a consequence of the fluid motion. Then, the power loss due to the gas warming, transferred by convection, can be analyzed by means of Newton's law of cooling: $P=h\left(T_{\mathrm{s}}\right.$ $\left.-T_{0}\right) 2 \pi r_{\mathrm{i}} L$, where the convection coefficient $h$ is introduced. The power loss, according to the model, is

$$
P=\left.\int_{0}^{L} \kappa \frac{\partial \Delta T}{\partial r}\right|_{r_{\mathrm{i}}} 2 \pi r_{\mathrm{i}} d z
$$

Thus

$h=\frac{\kappa}{L} \sum_{n=0}^{\infty} A_{n}\left[1-\exp \left(-\frac{\theta_{n}^{2} \kappa L}{\rho_{0} v_{0} C_{\mathrm{p}}}\right)\right]\left(\frac{\rho_{0} v_{0} C_{\mathrm{p}}}{\theta_{n} \kappa}\right)\left[J_{1}\left(\theta_{n} r_{\mathrm{i}}\right)-\alpha_{n} Y_{1}\left(\theta_{n} r_{\mathrm{i}}\right)\right]$

From Eq. 6 and 8 the dependences of thermal variables on the parameters that define the fluid regime can be derived.

Mass transport of species.- The growth of silicon in the seed rod can be either limited by chemical reactions or by mass transport. At a low temperature, the reaction rate is low and an excess of TCS is located in this region because few TCS molecules are converted into silicon and therefore the silicon growth is limited by the reaction. Otherwise, when the temperature rises, the reaction rate increases and there is a lack of TCS on the surface because there are no TCS molecules left to convert into silicon and, therefore, the silicon growth is limited by mass transport.

In the mass transport model presented below, both limitations can be analyzed. The only chemical reaction considered is the silicon deposition, although some gas phase reactions may appear, promoted by high temperatures in the gas phase; for instance, the conversion of $\mathrm{HSiCl}_{3}$ into $\mathrm{SiCl}_{4}{ }^{10}$ or the homogeneous nucleation of condensed silicon. The latter can be disregarded in the case of $\mathrm{HSiCl}_{3}$ and $T_{\mathrm{s}} \simeq 1400 \mathrm{~K}$. ${ }^{11}$ The $\mathrm{HSiCl}_{3}$ conversion into $\mathrm{SiCl}_{4}$ can also be disregarded because the growth rate is limited in a wide range of gas compositions by the hydrogen concentration at the rod surface.

The individual mass balance of species $i$ in the gas is

$$
\rho v_{z} \frac{\partial w_{i}}{\partial z}=\frac{1}{r} \frac{\partial}{\partial r}\left(\rho D_{i} r \frac{\partial w_{i}}{\partial r}\right)
$$

The following silicon deposition overall reaction has been considered $^{12}$

$$
\mathrm{HSiCl}_{3}+\mathrm{H}_{2} \rightarrow \mathrm{Si}+3 \mathrm{HCl}
$$

The incorporation of silicon atoms into the crystal lattice in polysilicon is supposed to be that of the single-crystalline silicon, because the growth rate does not vary considerably with different crystal orientations. ${ }^{13}$ The reaction is assumed to be a second-order reaction, and the rate of mass consumption or generation of species $i$ on the surface is expressed as

$$
R_{\mathrm{i}}=v_{\mathrm{i}} M_{\mathrm{i}} k\left[\mathrm{HSiCl}_{3}\right]\left[\mathrm{H}_{2}\right] \quad\left(\mathrm{kg} \mathrm{m}^{-2} \mathrm{~s}^{-1}\right)
$$

where $k$ is the overall constant reaction, $\nu_{\mathrm{i}}$ is the stoichiometry coefficients of involved compounds $\left(-1\right.$ for $\mathrm{HSiCl}_{3}$ and $\mathrm{H}_{2}$ and 3 for 
$\mathrm{HCl}$ ), and $[i]$ the mole concentration of species $i$ on the surface. A boundary condition for Eq. 9 will set that this rate of mass consumption or generation is equal to the diffusion flux driven by the concentration. The overall reaction constant is analyzed in Ref. 12 and depends on the rate of $\mathrm{HSiCl}_{3}$ chemisorption on the surface, $k_{\mathrm{ad}}$, and the rate of decomposition into $\mathrm{Si}, k_{\mathrm{r}}$. It can be expressed as

$$
\frac{1}{k}=\frac{\left[\mathrm{HSiCl}_{3}\right]}{k_{\mathrm{r}}}+\frac{\left[\mathrm{H}_{2}\right]}{k_{\mathrm{ad}}}
$$

Both rate constants obey Arrhenius's law and can be expressed at atmospheric pressure as

$$
\begin{aligned}
& k_{\text {ad }}(T)=2.72 \times 10^{6} \exp \left(\frac{-1.72 \times 10^{5}}{R T}\right) \\
& k_{\mathrm{r}}(T)=5.63 \times 10^{3} \exp \left(\frac{-1.80 \times 10^{5}}{R T}\right)
\end{aligned}
$$

In order to aid in the solution of the system, two extreme situations have been considered, as follows

$$
\begin{aligned}
\frac{\left[\mathrm{HSiCl}_{3}\right]}{k_{\mathrm{r}}} & >\frac{\left[\mathrm{H}_{2}\right]}{k_{\mathrm{ad}}} \Rightarrow k\left[\mathrm{HSiCl}_{3}\right]\left[\mathrm{H}_{2}\right] \simeq k_{\mathrm{r}} \cdot\left[\mathrm{H}_{2}\right] \\
& \Rightarrow \text { growth limited by } \mathrm{H}_{2} \\
\frac{\left[\mathrm{HSiCl}_{3}\right]}{k_{\mathrm{r}}}< & \frac{\left[\mathrm{H}_{2}\right]}{k_{\mathrm{ad}}} \Rightarrow k\left[\mathrm{HSiCl}_{3}\right]\left[\mathrm{H}_{2}\right] \simeq k_{\mathrm{ad}} \cdot\left[\mathrm{HSiCl}_{3}\right] \\
\Rightarrow & \text { growth limited by } \mathrm{HSiCl}_{3}
\end{aligned}
$$

This approach, based on the different orders of magnitude of both constants, allows a second-order reaction CVD system to be converted into a first-order reaction system, suitable to be solved analytically. The limitation changes along the silicon rod when concentration of $\mathrm{HSiCl}_{3}$ and $\mathrm{H}_{2}$ at the surface fulfills the following expression

$$
\frac{\left[\mathrm{HSiCl}_{3}\right]}{k_{\mathrm{r}}}=\frac{\left[\mathrm{H}_{2}\right]}{k_{\mathrm{ad}}} \Leftrightarrow \frac{\rho_{\mathrm{s}} w_{\mathrm{TCS}}}{M_{\mathrm{TCS}} k_{\mathrm{r}}}=\frac{\rho_{\mathrm{s}} w_{\mathrm{H}_{2}}}{M_{\mathrm{H}_{2}} k_{\mathrm{ad}}} \Leftrightarrow w_{\mathrm{TCS}}=\frac{k_{\mathrm{r}}}{k_{\mathrm{ad}}} \frac{M_{\mathrm{TCS}}}{M_{\mathrm{H}_{2}}} w_{\mathrm{H}_{2}}
$$

Growth limited by $\mathrm{H}_{2}$.- At the entrance of the reactor, the mass fraction of species is constant along the radius, and only $\mathrm{HSiCl}_{3}$ and $\mathrm{H}_{2}$ exist because the $\mathrm{HCl}$ has not been generated yet. Then, it can be stated that the deposition process begins limited by $\mathrm{H}_{2}$, taking into account that $w_{\mathrm{TCS}_{0}}=1-w_{\mathrm{H}_{20}}$, when

$$
w_{\mathrm{H}_{20}} \leqslant \frac{1}{1+\left(k_{\mathrm{r}} / k_{\mathrm{ad}}\right)\left(M_{\mathrm{TCS}} / M_{\mathrm{H}_{2}}\right)}
$$

The system that has to be solved is the corresponding partial derivative equation (Eq. 9) for each compound: trichlorosilane, hydrogen, and hydrochloric acid, respectively, and the following boundary conditions: (i) mass fraction at the entrance, known and constant; (ii) closed boundary at $r_{\mathrm{o}}$; and (iii) the diffusion flux driven by the concentration is equal to the rate of mass consumption or generation at $r_{\mathrm{i}}$.

When growth rate is limited by $\mathrm{H}_{2}$, the boundary conditions for the mass transport equation are as follows

$$
\begin{gathered}
\left.w_{i}\right|_{z=0}=w_{i 0} \\
\left.\frac{\partial w_{i}}{\partial r}\right|_{r_{\mathrm{o}}}=0 \\
\left.\rho_{0} D_{i_{0}} \frac{\partial w_{i}}{\partial r}\right|_{r_{\mathrm{i}}}=-\left.v_{i} M_{i} \rho_{\mathrm{s}} k_{\mathrm{r}}\left(T_{\mathrm{s}}\right) \frac{1}{M_{\mathrm{H}_{2}}} w_{\mathrm{H}_{2}}\right|_{r_{\mathrm{i}}}
\end{gathered}
$$

$$
i=\mathrm{HSiCl}_{3}, \quad \mathrm{H}_{2}, \quad \mathrm{HCl}
$$

Equation 9 for hydrogen leads to the following solution (see Appendix $\mathrm{B}$ for its derivation)

$$
w_{\mathrm{H}_{2}}(r, z)=w_{\mathrm{H}_{20}} \sum_{n=0}^{\infty} A_{n} \exp \left(-\frac{\theta_{n}^{2} D_{1_{0}} z}{v_{0}}\right) \beta_{n}(r)
$$

Note that, due to mass fraction definition: $w_{\mathrm{HCl}}=1-w_{\mathrm{TCS}}-w_{\mathrm{H}_{2}}$, and as can be seen from the equations system

$$
w_{\mathrm{TCS}}-w_{\mathrm{TCS}_{0}}=\frac{M_{\mathrm{TCS}}}{M_{\mathrm{H}_{2}}}\left(w_{\mathrm{H}_{2}}-w_{\mathrm{H}_{20}}\right) \quad \forall r, z
$$

The growth rate is derived from Eq. 11, considering that the growth is limited by $\mathrm{H}_{2}$

$$
v_{\mathrm{g}}(z)=\frac{6 \times 10^{7}}{\rho_{\mathrm{Si}}} \frac{M_{\mathrm{Si}}}{M_{\mathrm{H}_{2}}} \rho_{\mathrm{s}} k_{\mathrm{r}}\left(T_{\mathrm{s}}\right) w_{\mathrm{H}_{2}}\left(r_{\mathrm{i}}, z\right) \quad(\mu \mathrm{m} / \mathrm{min})
$$

where $\rho_{\mathrm{Si}}$ is the density of solid silicon expressed in $\mathrm{kgm}^{-3}$. The mass fraction of $\mathrm{HSiCl}_{3}$ and $\mathrm{H}_{2}$ diminishes along the rod length, whereas the $\mathrm{HCl}$ mass fraction increases. Even though the growth is limited by $\mathrm{H}_{2}$, the situation changes when $w_{\mathrm{TCS}}$ and $w_{\mathrm{H}_{2}}$ on the rod surface meets Eq. 15. Also, taking into account Eq. 21, the value of $w_{\mathrm{H}_{2}}$ on the rod surface when the limitation changes is

$$
w_{\mathrm{H}_{2}}\left(r_{\mathrm{i}}, z_{1}\right)=w_{\mathrm{H}_{2} \lim }=\frac{\left(1+\frac{M_{\mathrm{TCS}}}{M_{\mathrm{H}_{2}}}\right) w_{\mathrm{H}_{20}}-1}{\left(M_{\mathrm{TCS}} / M_{\mathrm{H}_{2}}\right)\left(1-\frac{k_{\mathrm{r}}}{k_{\mathrm{ad}}}\right)} \leqslant w_{\mathrm{H}_{20}}
$$

The height of the rod where the limitation changes is $z_{1}$, i.e., $w_{\mathrm{H}_{2}}$ $\left(r_{\mathrm{i}}, z_{\mathrm{l}}\right)=w_{\mathrm{H}_{2 \lim }}=w_{\mathrm{H}_{z_{2}}}$.

Limitation change.- The reaction rate is, at this height of the rod, limited by $\mathrm{HSiCl}_{3}$ chemisorption on the surface. This situation has to be considered when $w_{\mathrm{H}_{20}}$ meets the conditions: $w_{\mathrm{H}_{2 l i m}} \geqslant 0$ and Eq. 16, i.e.

$$
\frac{M_{\mathrm{H}_{2}}}{M_{\mathrm{H}_{2}}+M_{\mathrm{TCS}}} \leqslant w_{\mathrm{H}_{20}} \leqslant \frac{1}{1+\left(k_{\mathrm{r}} / k_{\mathrm{ad}}\right)\left(M_{\mathrm{TCS}} / M_{\mathrm{H}_{2}}\right)}
$$

The boundary conditions must change because the growth is now limited by $\mathrm{HSiCl}_{3}$. The new conditions that define the system are as follows

$$
\begin{gathered}
\left.w_{i}\right|_{z=z_{1}}=w_{i_{z 1}} \\
\left.\frac{\partial w_{i}}{\partial r}\right|_{r_{\mathrm{o}}}=0 \\
\left.\rho_{0} D_{i_{0}} \frac{\partial w_{i}}{\partial r}\right|_{r_{\mathrm{i}}}=-\left.v_{i} M_{i} \rho_{\mathrm{s}} k_{\mathrm{ad}}\left(T_{\mathrm{s}}\right) \frac{1}{M_{\mathrm{TCS}}} w_{\mathrm{TCS}}\right|_{r_{\mathrm{i}}} \\
i=\mathrm{HSiCl}_{3}, \quad \mathrm{H}_{2}, \quad \mathrm{HCl}
\end{gathered}
$$

The equation for $\mathrm{HSiCl}_{3}$ is solved and its mass fraction is obtained by means of the following summatory (see Appendix C for its derivation)

$$
w_{\mathrm{TCS}}(r, z)=\sum_{n=0}^{\infty} B_{n} \exp \left(-\frac{\eta_{n}^{2} D_{1_{0}}\left(z-z_{1}\right)}{v_{0}}\right) \delta_{n}(r) \quad \forall z>z_{1}
$$

Again, due to mass fraction definition in the case of $w_{\mathrm{HCl}}$ and due to the equations system, it can be derived that

$$
w_{\mathrm{H}_{2}}-w_{\mathrm{H}_{20}}=\frac{M_{\mathrm{H}_{2}}}{M_{\mathrm{TCS}}}\left(w_{\mathrm{TCS}}-w_{\mathrm{TCS}_{0}}\right) \quad \forall r, \quad \forall z>z_{1}
$$




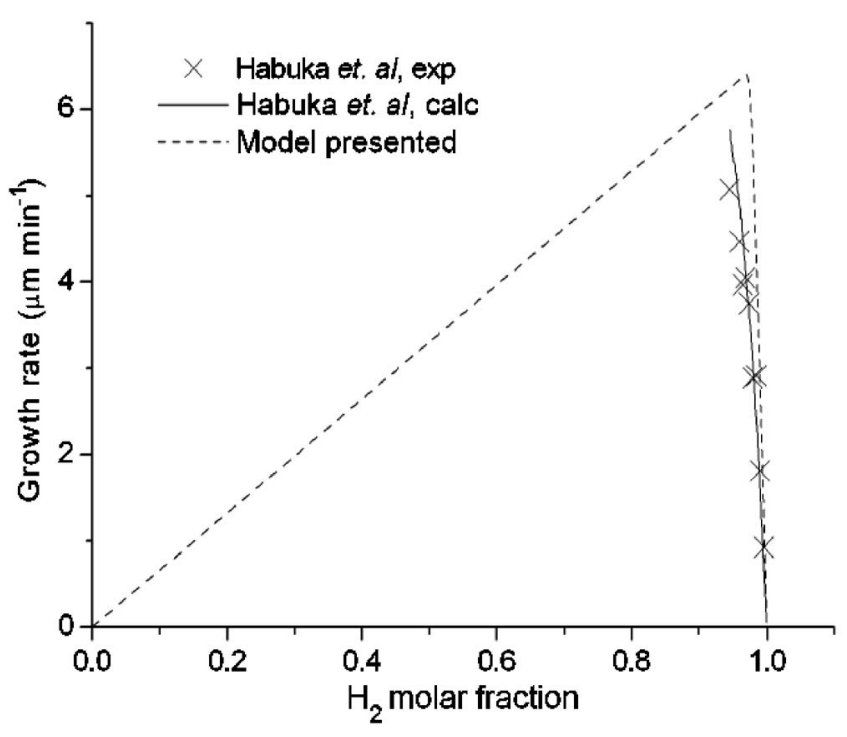

Figure 2. Growth rates obtained from the model compared to those presented in the literature. Calculated in Ref. $12(-)$, measured in Ref. 12 (X), and model presented $(\cdots)$.

$$
w_{\mathrm{HCl}}=1-w_{\mathrm{H}_{2}}-w_{\mathrm{TCS}} \quad \forall r, \quad \forall z>z_{1}
$$

The growth rate is expressed as

$$
v_{\mathrm{g}}(z)=\frac{6 \times 10^{7}}{\rho_{\mathrm{Si}}} \frac{M_{\mathrm{Si}}}{M_{\mathrm{TCS}}} \rho_{\mathrm{s}} k_{\mathrm{ad}}\left(T_{\mathrm{s}}\right) w_{\mathrm{TCS}}\left(r_{\mathrm{i}}, z\right) \quad \forall z>z_{1} \quad(\mu \mathrm{m} / \mathrm{min})
$$

Growth limited by $\mathrm{HSiCl}_{3}$. - In this situation, the growth process begins limited by $\mathrm{HSiCl}_{3}$, i.e.

$$
w_{\mathrm{H}_{20}}>\frac{1}{1+\left(k_{\mathrm{r}} / k_{\mathrm{ad}}\right)\left(M_{\mathrm{TCS}} / M_{\mathrm{H}_{2}}\right)}
$$

The solution of this system is similar to that presented in Eq. 20-22, taking into account that $k_{\text {ad }}$ has to be used instead of $k_{\mathrm{r}}$ and $w_{\mathrm{TCS}}$ plays the role of $w_{\mathrm{H}_{2}}$ and vice versa. In this case, $w_{\mathrm{H}_{20}}<w_{\mathrm{H}_{2 \lim }}$ and then the evolution of $w_{\mathrm{H}_{2}}$ never reaches the changing limitation value. Thus, it can be assured that the growth remains limited by $\mathrm{HSiCl}_{3}$ and does not change to limited by $\mathrm{H}_{2}$.

\section{Comparison of the Model to Published Data}

The model for the deposition process has been compared to experimental and calculated data obtained from the literature. The most complete study corresponds to Habuka et al., ${ }^{12}$ where the deposition takes place on a $20 \mathrm{~cm}$ diam wafer held horizontally in a reactor whose walls are separated $20 \mathrm{~mm}$ from the substrate. The system geometry presented in Ref. 12 does not exactly match the system presented in this study, which analyzes the deposition process of $\mathrm{HSiCl}_{3}$ and $\mathrm{H}_{2}$ on a silicon rod seed. The following analysis has been carried out in order to make both systems as similar as possible: rod length, $20 \mathrm{~cm}$; rod diameter, $20 \mathrm{~cm}$; and wall diameter, $22 \mathrm{~cm}$. The gas velocity is $v=0.67 \mathrm{~ms}^{-1}$, the surface temperature is $T_{\mathrm{S}}=1398 \mathrm{~K}$, and the pressure is atmospheric. The comparison between the average growth rate over the substrate is presented in Fig. 2. It can be derived that the model predicts the growth rate.

\section{Model Results}

Some reactor conditions are considered before beginning the analysis: $T_{0}=773 \mathrm{~K}, T_{\mathrm{s}}=1423 \mathrm{~K}, p=1 \mathrm{~atm}, L=1.5 \mathrm{~m}, r_{\mathrm{o}}$ $=15 \mathrm{~cm}$, and $r_{\mathrm{i}}=5 \mathrm{~cm}$. The values of $v$ and $x_{\mathrm{H}_{20}}$ that set an average temperature at the outlet of $900 \mathrm{~K}$ have been obtained. $x_{\mathrm{H}_{20}}$ is the molar fraction of hydrogen at the inlet. For these values, the growth

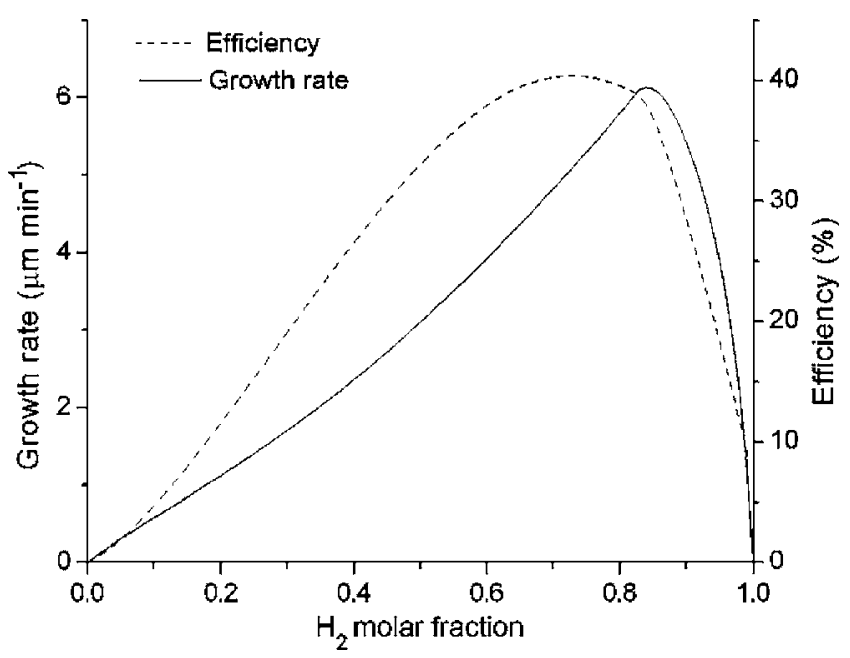

Figure 3. Growth rate (-) and deposition efficiency (- -). The average temperature at the outlet is $900 \mathrm{~K}$.

rate average, the deposition efficiency (rate between silicon deposited and silicon introduced), and the convection coefficient are calculated and presented in Fig. 3 and 4. The growth rate increases when raising the $\mathrm{HSiCl}_{3}$ molar fraction at the inlet from zero to a certain value, in this particular case $x_{\mathrm{TCS}_{0}}=0.17$, then it decreases because of the hydrogen limitation. This maximum depends on the flow regime, the surface temperature, the temperature of the gas mixture at the inlet, or even the pressure within the reactor vessel. The efficiency reaches a maximum at a slightly different $\mathrm{HSiCl}_{3}$ molar fraction, which is always higher. Although the power loss because of convection is not as important as the radiation loss, ${ }^{14}$ it can be seen that it increases fivefold when the hydrogen molar fraction at the inlet is $>0.95$.

Effect of gas velocity.- The evolution of the deposition features keeping $x_{\mathrm{H}_{20}}$ constant and modifying the gas velocity from $\mathrm{Re}$ $=1-2300$, which is the limit considered for laminar flow, has been analyzed. This analysis is detailed in Fig. 5. When the velocity is low, the rate of silicon introduced in the reactor per unit of time is low and, therefore, it can be consumed throughout the entire length

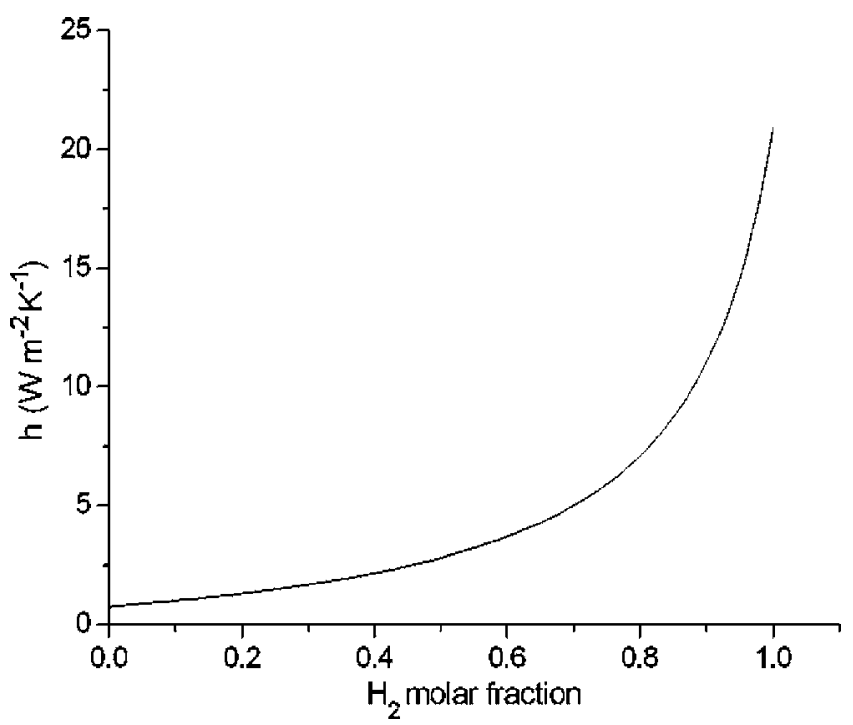

Figure 4. Convection coefficient under the same gas conditions analyzed in Fig. 3. 


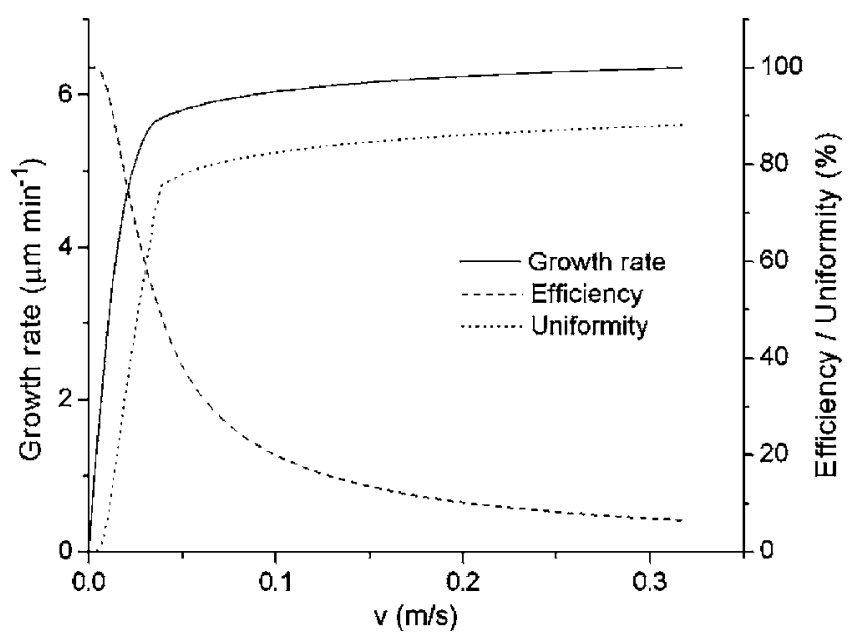

Figure 5. Growth rate (-), uniformity $(\cdots)$, and deposition efficiency (- -) for different gas velocities at the inlet, from $\mathrm{Re}=1-2300$. Gas molar composition: $x_{\mathrm{h}_{20}}=0.8$.

of the reactor yielding high efficiencies. But, low velocities generate an even, mass fraction profile yielding low growth rates due to the low diffusion of mass toward the silicon surface. The growth uniformity, defined as $v_{\mathrm{g}}(L) / v_{\mathrm{g}}(0) \times 100$, is also analyzed. It can be seen that by increasing the gas velocity, the growth uniformity along the rod also increases. The flow regime determined by $R e=2300$ gives the maximum growth rate and the minimum deposition efficiency for a given gas composition. The velocity of the gas can be chosen according to several criteria: high growth rate, high deposition efficiency, low power loss, high growth uniformity, or low average temperature at the outlet. The analysis presented provides valuable information to make a decision.

Effect of the rod surface temperature.- A lot of information on the optimum deposition temperature can be obtained from Ref. 12 and 15. The analysis carried out consists of obtaining the growth rate, considering three different rod surface temperatures: 1373, 1423 , and $1500 \mathrm{~K}$. The flow regime is $\mathrm{Re}=2300$ at the inlet, in order to find out the maximum growth rate for every gas composition. Arrhenius's law, which defines the reaction constants, is very temperature sensitive and thus a linear reduction of $T_{\mathrm{s}}$ yields an exponential reduction of the growth rate. The higher the temperature is, the higher the growth rate is, as can be seen in Fig. 6. The minimum deposition efficiency has the same dependence on the surface temperature. The information provided by the model does not impose any limit on the temperature on the rod surface, but it exists. The analysis carried out in Ref. 14 shows that around 1400-1450 K is an appropriate rod temperature because higher temperatures introduce thermal stresses in the rod or allows the center of the rod to reach the silicon melting temperature, $1687 \mathrm{~K}$.

Effect of the gas inlet temperature.-Several reasons suggest warming the gas mixture before introducing it into the reactor, for instance, to take advantage of the heat of the exhaust gases or to reduce the cooling effect of the gases flowing across the silicon rod. One more is proposed in this paper: to increase the growth rate. The effect of the gas inlet temperature on the deposition rate is analyzed, and the results can be observed in Fig. 7. Three different temperatures are considered: 300,523 , and $773 \mathrm{~K}$, where the flow regime is determined by $\mathrm{Re}=2300$ at the inlet. It can be seen that the maximum growth rate increases due to higher binary diffusion coefficient and shifts to higher $\mathrm{H}_{2}$ molar fraction when the temperature is raised. Despite increasing the growth rate, the deposition efficiency decreases with temperature because the amount of silicon introduced into the reactor is higher when the gas inlet temperature is raised (note that $\operatorname{Re}=2300$ ).

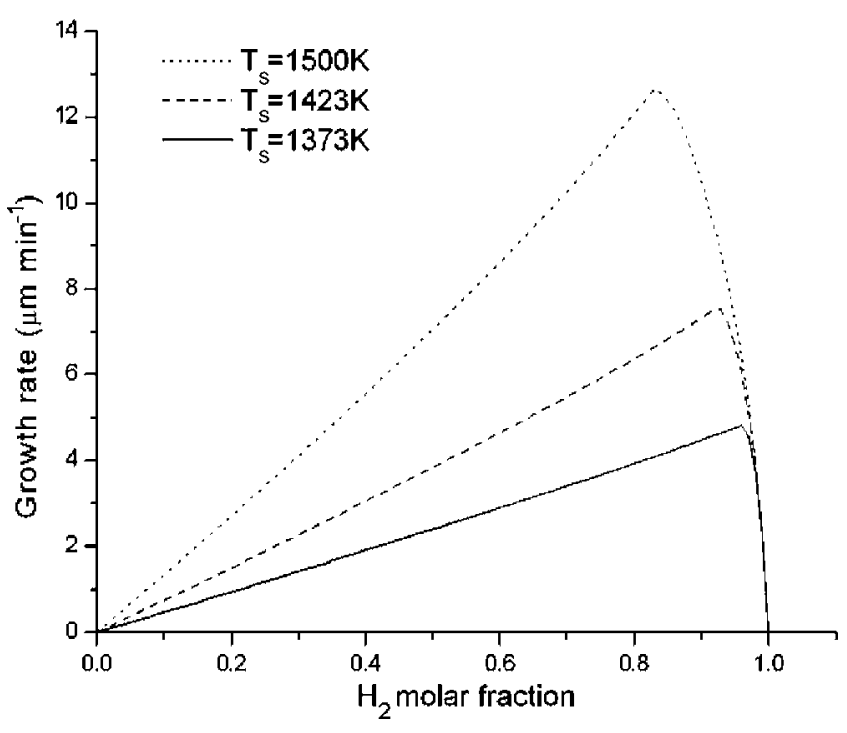

Figure 6. Growth rate regarding different rod surface temperatures: (-) $T_{\mathrm{s}}=1373 \mathrm{~K}$; and $(--) T_{\mathrm{s}}=1423 \mathrm{~K} ;(\cdots) T_{\mathrm{s}}=1500 \mathrm{~K} . \mathrm{Re}=2300$ at the inlet.

Effect of pressure.- It has to be understood that the reaction constants presented in Eq. 13 and 14 are validated for atmospheric pressure, and therefore, they should not be used to evaluate the effect of high or low pressure inside the reactor vessel. Nevertheless, it is considered that the analysis can provide some trend or information. Thus, the effect of increasing the pressure in the reactor has been analyzed and the results are detailed in Fig. 8. The growth rate increases with the pressure because the concentration of the gas, i.e., the reaction precursors, is higher. The maximum growth rate shifts to a higher $\mathrm{H}_{2}$ molar fraction. The minimum deposition efficiency has the same dependence on the pressure as the growth rate. To operate the reactor at high pressure can diminish the deposition time, lowering the energy consumed during the process even though the convection coefficient increases.

\section{Discussion}

Two different approaches are usually proposed regarding growth rate and deposition efficiency: to seek the deposition conditions that

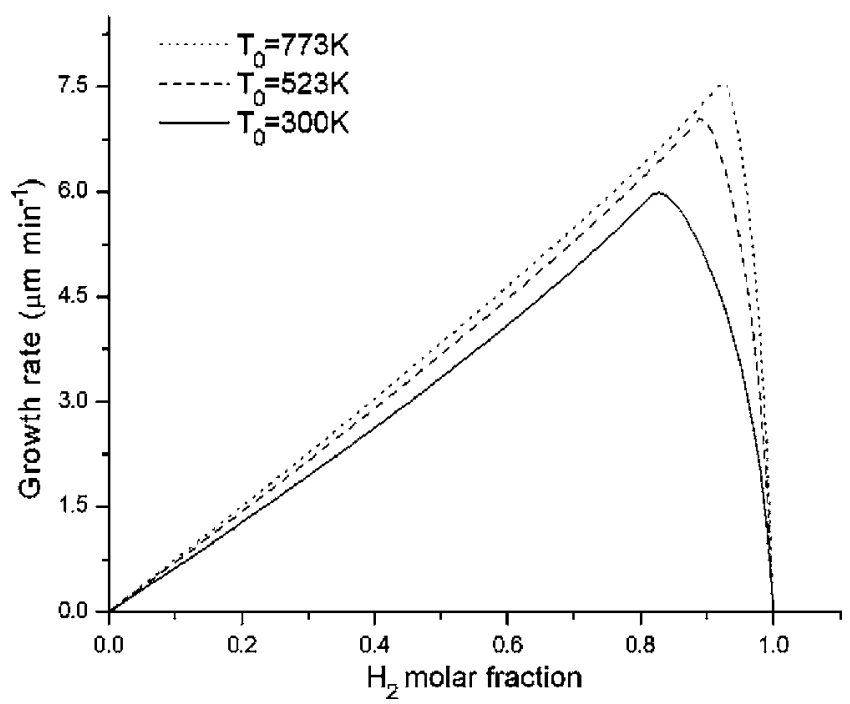

Figure 7. Growth rate regarding different gas inlet temperatures: (-) $T_{0}$ $=300 \mathrm{~K}$, and $(--) T_{0}=523 \mathrm{~K},(\cdots) T_{0}=773 \mathrm{~K}$. Re $=2300$ at the inlet. 


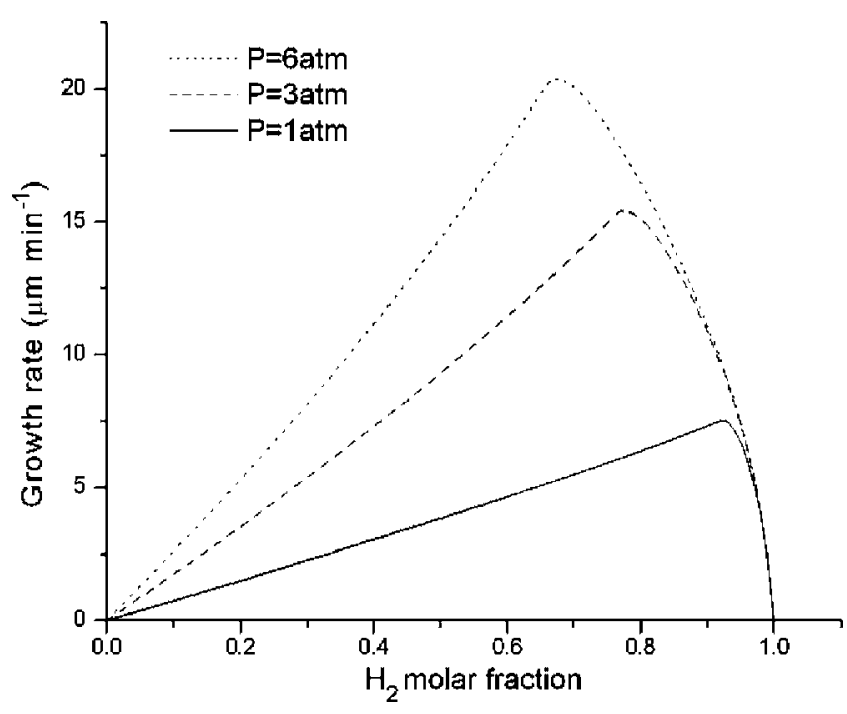

Figure 8. Growth rate regarding different reactor pressures: $(-) p$ $=1 \mathrm{~atm}$; and $(--) p=3 \mathrm{~atm} ;(\cdots) p=6 \mathrm{~atm} . \operatorname{Re}=2300$ at the inlet.

provides the maximum growth rate or to seek high deposition efficiencies. The former diminishes the energy consumption by lowering the operation time per polysilicon batch. The latter allows a smaller gas recovery system. A reduction in the energy consumption, and therefore operating at the maximum growth rate regime, is needed to reduce the cost of the polysilicon deposition technology, making solar grade silicon cheaper.

The rod surface temperature must always be $>1400 \mathrm{~K}$ because this is a very important parameter in the deposition process, as can be seen in Fig. 6. The gas inlet temperature should be raised to achieve higher growth rates and reduce the energy consumed due to rod cooling. An appropriate range of inlet temperature can be between 673 and $773 \mathrm{~K}$, because the growth rate increases without promoting gas phase reactions during this preheating process. By increasing the gas velocity at the inlet, the growth rate rises until a certain value at which a flat growth rate profile is obtained and the deposition efficiency decreases. At the beginning of this flat profile, a trade-off between high growth rate and good deposition efficiency can be achieved, as can be seen in Fig. 5. This flat growth rate profile diminishes when shifting to a lower molar fraction of $\mathrm{HSiCl}_{3}$ at the inlet, increasing the gas velocity required to obtain the maximum growth rate. At atmospheric pressure, a good composition at the inlet could be $x_{\mathrm{H}_{2}}=0.85$, with a gas velocity close to $v$ $=0.1 \mathrm{~ms}^{-1}$.

Operation above atmospheric pressure is strongly recommended, according to data shown in Fig. 8, because it provides higher deposition rates and, therefore, reduces the batch deposition time. Growth rates of $20 \mu \mathrm{m} \mathrm{min}{ }^{-1}$ are estimated when raising the pressure of the reactor to $6 \mathrm{~atm}$.

\section{Conclusions}

A chemical deposition model is required to analyze the cost of the polysilicon deposition process or its environmental impact, aiming at maximizing the rate between the amount of polysilicon obtained and the energy consumed throughout the deposition process. By means of the approach presented in this paper, the conversion of the second-order reaction rate into two systems of first-order reaction rate, an analytical chemical deposition model is presented.

The dependence of the growth features on the gas velocity and on the gas composition at the inlet is predicted by the model, providing valuable information on the deposition efficiency, the growth rate, and the uniformity. The effect of the reactor pressure and the rod surface temperature is also understood and presented.
At atmospheric pressure, the composition at the inlet should be between $x_{\mathrm{H}_{2}}=0.85$ and 0.90 , with a gas velocity of around Re $=800$. The gas temperature at the inlet should be raised to the range between 673 and $773 \mathrm{~K}$. The resulting growth rate will be between 6 and $6.5 \mu \mathrm{m} \mathrm{min}^{-1}$. The geometry of the system is presented in Fig. 1.

Operation above atmospheric pressure is strongly recommended, and the model predicts growth rates of $20 \mu \mathrm{m} \mathrm{min}^{-1}$ at $6 \mathrm{~atm}$. The deposition time is reduced by almost four times while the power loss is similar. Thus, energy consumption is reduced fourfold.

\section{Acknowledgment}

This work has been partially funded by the Spanish Ministerio de Educación y Ciencia, through project no. PSE-120000-2006-2.

Universidad Politécnica de Madrid assisted in meeting the publication costs of this article.

\section{Appendix A: Temperature Distribution}

The energy conservation equation is used to obtain the temperature distribution

$$
\rho C_{\mathrm{p}} v_{z} \frac{\partial T}{\partial z}=\frac{1}{r} \frac{\partial}{\partial r}\left(\kappa r \frac{\partial T}{\partial r}\right)
$$

In Eq. A-1 the variation of thermal conductivity with temperature is ignored and can be derived from ${ }^{6,7}$

$$
\left.\begin{array}{l}
\kappa \simeq \kappa_{0}\left(\frac{T}{T_{0}}\right)^{0.5+0.14874}=\kappa_{0}\left(\frac{T}{T_{0}}\right)^{0.64874} \\
\frac{d \kappa}{d T} \simeq \frac{\kappa_{0}}{T_{0}}\left(\frac{T}{T_{0}}\right)^{0.64874-1}
\end{array}\right\} \frac{d \kappa}{d T} \leqslant \frac{1}{T_{0}} \kappa
$$

where $T_{0}$ is the gas temperature at the entrance of the reactor, whose value is fixed at $T_{0}=773 \mathrm{~K}$. It is shown that the thermal conductivity is $\sim 773$ times greater than its derivative, and therefore, the latter can be ignored in the equation and the former can be outside the derivative. According to Eq. 4 and A-2, Eq. A-1 can be expressed as

$$
\rho_{0} C_{\mathrm{p}} v_{0} \frac{\partial \Delta T}{\partial z}=\kappa \frac{1}{r} \frac{\partial}{\partial r}\left(r \frac{\partial \Delta T}{\partial r}\right)
$$

where $\Delta T(r, z)=T(r, z)-T_{\mathrm{s}}$. The separation of variables method applied to Eq. A-3 leads to

$$
\Delta T(r, z)=\exp \left(-\frac{\theta^{2} \kappa z}{\rho_{0} v_{0} C_{\mathrm{p}}}\right)\left[A J_{0}(\theta r)+B Y_{0}(\theta r)\right]
$$

$J_{0}$ and $Y_{0}$ are the first- and second-kind Bessel functions of order zero, respectively. The boundary conditions for $\Delta T$ are: $(i)$ the temperature of the gas on the rod surface is $T_{\mathrm{s}}$, $\Delta T\left(r_{\mathrm{i}}, z\right)=0$, (ii) adiabatic condition in $r_{\mathrm{o}} \partial \Delta T / \partial r\left(r_{\mathrm{o}}, z\right)=0$, and (iii) constant and known temperature distribution at the reactor entrance, $\Delta T(r, 0)=\Delta T_{0}$. Conditions $(i)$ and (ii) yield a relation that $\theta$ has to fulfill to be part of the solution of the problem

$$
J_{0}\left(\theta r_{\mathrm{i}}\right) Y_{1}\left(\theta r_{\mathrm{o}}\right)-J_{1}\left(\theta r_{\mathrm{o}}\right) Y_{0}\left(\theta r_{\mathrm{i}}\right)=0
$$

The set of solutions of this equation is $\left\{\theta_{n}\right\}$. Condition (iii) leads to the solution by means of the following

$$
\Delta T(r, z)=\Delta T_{0} \sum_{n=0}^{\infty} A_{n} \exp \left(-\frac{\theta_{n}^{2} \kappa z}{\rho_{0} v_{0} C_{\mathrm{p}}}\right) \chi_{n}(r)
$$

$A_{n}$ is the coefficient corresponding to the orthogonal function $\chi_{n}$, calculated following the Sturm-Liouville conditions, and $\kappa$ is the thermal conductivity at $T_{\mathrm{m}}=\left(T_{\mathrm{s}}-T_{0}\right) / 2$

$$
\begin{aligned}
& \chi_{n}(r)=\left[J_{0}\left(\theta_{n} r\right)-\frac{J_{0}\left(\theta_{n} r_{\mathrm{i}}\right)}{Y_{0}\left(\theta_{n} r_{\mathrm{i}}\right)} Y_{0}\left(\theta_{n} r\right)\right]=\left[J_{0}\left(\theta_{n} r\right)-\alpha_{n} Y_{0}\left(\theta_{n} r\right)\right] \\
& A_{n}=\frac{\int_{r_{\mathrm{i}}}^{r_{\mathrm{i}}} \chi_{n}(r) d r}{\int_{r_{\mathrm{i}}}^{r_{\mathrm{o}}} r \chi_{n}^{2}(r) d r}=\frac{-\frac{r_{i}}{\theta_{n}}\left[J_{1}\left(\theta_{n} r_{\mathrm{i}}\right)-\alpha_{n} Y_{1}\left(\theta_{n} r_{\mathrm{i}}\right)\right]}{\frac{1}{2}\left\{r_{\mathrm{o}}^{2} \chi_{n}\left(r_{\mathrm{o}}\right)^{2}-r_{\mathrm{i}}^{2}\left[J_{1}\left(\theta_{n} r_{\mathrm{i}}\right)-\alpha_{n} Y_{1}\left(\theta_{n} r_{\mathrm{i}}\right)\right]\right\}}
\end{aligned}
$$

\section{Appendix B: Mass Fraction Distribution, Growth Limited by $\mathrm{H}_{2}$}

The individual mass balance of species $i$ in the gas is

$$
\rho v_{z} \frac{\partial w_{i}}{\partial z}=\frac{1}{r} \frac{\partial}{\partial r}\left(\rho D_{i} r \frac{\partial w_{i}}{\partial r}\right)
$$




$$
\left.\begin{array}{l}
\rho D_{i} \simeq \rho_{0} D_{i 0}\left(\frac{T}{T_{0}}\right)^{0.6561} \\
\frac{d \rho D_{i}}{d T} \simeq \frac{\rho_{0} D_{i 0}}{T_{0}}\left(\frac{T}{T_{0}}\right)^{0.6561-1}
\end{array}\right\} \frac{d \rho D_{i}}{d T} \leqslant \frac{1}{T_{0}} \rho D_{i}
$$

Then, taking into account Eq. 4 and B-2, the partial derivative equation yields

$$
\frac{v_{0}}{D_{i 0}} \frac{\partial w_{i}}{\partial z}=\frac{1}{r} \frac{\partial}{\partial r}\left(r \frac{\partial w_{i}}{\partial r}\right)
$$

It has to be noted that $D_{i_{0}}$ stands for the binary diffusion coefficient at the inlet temperature between $\mathrm{HSiCl}_{3}$ and $\mathrm{H}_{2}$ in the case of $i=\mathrm{HSiCl}_{3}, \mathrm{H}_{2}$ and between $\mathrm{HCl}$ and $\mathrm{H}_{2}$ in the case of $i=\mathrm{HCl}$. The equation system is solved for hydrogen, with its boundary conditions shown in Eq. 17-19: (i) mass fraction at the entrance: known and constant, (ii) closed boundary at $r_{\mathrm{o}}$, and (iii) the diffusion flux driven by the concentration is equal to the rate of mass consumption or generation at $r_{\mathrm{i}}$. tion

The separation of variables method applied to Eq. B-3 leads to the following solu-

$$
w_{\mathrm{H}_{2}}(r, z)=w_{\mathrm{H}_{20}} \sum_{n=0}^{\infty} A_{n} \exp \left(-\frac{\theta_{n}^{2} D_{1_{0}} z}{v_{0}}\right) \beta_{n}(r)
$$

where $\left\{\theta_{n}\right\}$ is the set of solutions of Eq. B-5 and $A_{n}$ is the coefficient corresponding to the orthogonal functions $\beta_{n}$, calculated following the Sturm-Liouville conditions

$$
\begin{gathered}
{\left[\frac{\rho_{\mathrm{s}} k_{\mathrm{r}}}{\rho_{0} D_{1_{0}}} Y_{0}\left(\theta r_{\mathrm{i}}\right)+\theta Y_{1}\left(\theta r_{\mathrm{i}}\right)\right] J_{1}\left(\theta r_{\mathrm{o}}\right)-\left[\frac{\rho_{\mathrm{s}} k_{\mathrm{r}}}{\rho_{0} D_{1_{0}}} J_{0}\left(\theta r_{\mathrm{i}}\right)+\theta J_{1}\left(\theta r_{\mathrm{i}}\right)\right] Y_{1}\left(\theta r_{\mathrm{o}}\right)=0} \\
\beta_{n}(r)=\left[J_{0}\left(\theta_{n} r\right)-\frac{J_{1}\left(\theta_{n} r_{\mathrm{o}}\right)}{Y_{1}\left(\theta_{n} r_{\mathrm{o}}\right)} Y_{0}\left(\theta_{n} r\right)\right]=\left[J_{0}\left(\theta_{n} r\right)-\gamma_{n} Y_{0}\left(\theta_{n} r\right)\right] \\
A_{n}=\frac{-\frac{r_{\mathrm{i}}}{\theta_{n}}\left[J_{1}\left(\theta_{n} r_{\mathrm{i}}\right)-\gamma_{n} Y_{1}\left(\theta_{n} r_{\mathrm{i}}\right)\right]}{(1 / 2)\left\{r_{\mathrm{o}}^{2} \beta_{n}\left(r_{\mathrm{o}}\right)^{2}-r_{\mathrm{i}}^{2} \beta_{n}\left(r_{\mathrm{i}}\right)^{2}-r_{\mathrm{i}}^{2}\left[J_{1}\left(\theta_{n} r_{\mathrm{i}}\right)-\gamma_{n} Y_{1}\left(\theta_{n} r_{i}\right)\right]\right\}}
\end{gathered}
$$

\section{Appendix C: Mass Fraction Distribution, Growth after Limitation Change}

The equation solved is B-3, presented in Appendix B. The limitation is considered to change in $z_{1}$, i.e., $w_{\mathrm{H}_{2}}\left(r_{\mathrm{i}}, z_{1}\right)=w_{\mathrm{H}_{2 l i m}}=w_{\mathrm{H}_{2}}$. The boundary conditions, presented in Eq. 25-27, are: (i) the mass fraction at the limitation point, $z_{1}$, is known, (ii) closed boundary at $r_{\mathrm{o}}$, and (iii) the diffusion flux driven by the concentration is equal to the rate of mass consumption or generation at $r_{\mathrm{i}}$.

The equation for $\mathrm{HSiCl}_{3}$ is solved, and its mass fraction is obtained by means of the following summatory

$$
w_{\mathrm{TCS}}(r, z)=\sum_{n=0}^{\infty} B_{n} \exp \left(-\frac{\eta_{n}^{2} D_{1_{0}}\left(z-z_{1}\right)}{v_{0}}\right) \delta_{n}(r) \quad \forall z>z_{l}
$$

where $\left\{\eta_{n}\right\}$ is the set of solutions to Eq. C-2 and $B_{n}$ are the coefficient corresponding to the orthogonal functions $\delta_{n}$

$$
\left[\frac{\rho_{\mathrm{s}} k_{\mathrm{ad}}}{\rho_{0} D_{1_{0}}} Y_{0}\left(\eta r_{\mathrm{i}}\right)+\eta Y_{1}\left(\eta r_{\mathrm{i}}\right)\right] J_{1}\left(\eta r_{\mathrm{o}}\right)-\left[\frac{\rho_{\mathrm{s}} k_{\mathrm{ad}}}{\rho_{0} D_{1_{0}}} J_{0}\left(\eta r_{\mathrm{i}}\right)+\eta J_{1}\left(\eta r_{\mathrm{i}}\right)\right] Y_{1}\left(\eta r_{\mathrm{o}}\right)=0
$$

$$
\partial_{n}(r)=\left[J_{0}\left(\eta_{n} r\right)-\frac{J_{1}\left(\eta_{n} r_{\mathrm{o}}\right)}{Y_{1}\left(\eta_{n} r_{\mathrm{o}}\right)} Y_{0}\left(\eta_{n} r\right)\right]=\left[J_{0}\left(\eta_{n} r\right)-\gamma_{n} Y_{0}\left(\eta_{n} r\right)\right]
$$

The method for obtaining $B_{n}$ is the same as for obtaining $A_{n}$, but there is one more integral involved that should be known

$$
\int_{r_{\mathrm{i}}}^{r_{\mathrm{o}}} r \beta_{m}(r) \delta_{n}(r) d r=-\frac{1}{\theta_{m}^{2}-\eta_{n}^{2}} \frac{\rho_{\mathrm{s}}}{\rho_{0} D_{1_{0}}}\left(k_{\mathrm{ad}}-k_{\mathrm{r}}\right) r_{\mathrm{i}} \beta_{m}\left(r_{\mathrm{i}}\right) \delta_{n}\left(r_{\mathrm{i}}\right) \quad \forall n, m
$$

\section{List of Symbols}

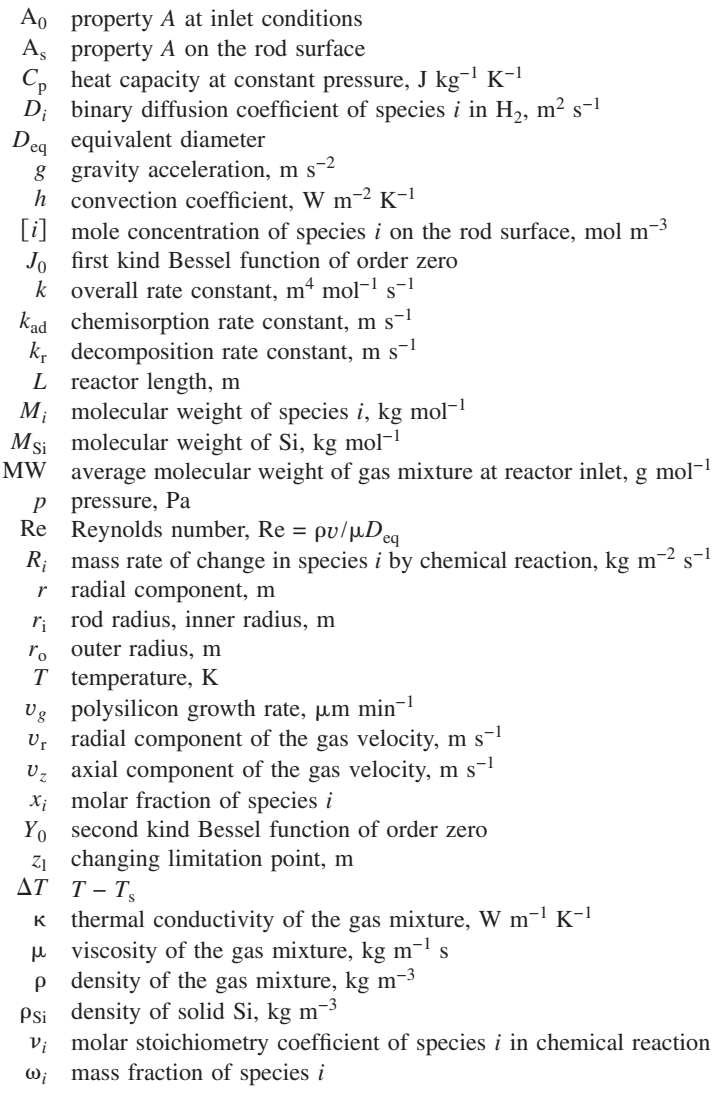

\section{References}

1. M. Rogol, Paper presented at the 3rd Solar Silicon Conference, April 1, 2006.

2. A. Mozer and P. Fath, Paper presented at the 3rd Solar Silicon Conference, April 1, 2006.

3. J. W. Ager, J. W. Beeman, W. L. Hansen, E. E. Haller, I. D. Sharp, C. Liao, A Yang, M. L. W. Thewalt, and H. Riemann, J. Electrochem. Soc., 152, 448 (2005).

4. K. Hashimoto, K. Miura, T. Masuda, M. Toma, H. Sawai, and M. Kawase, J. Electrochem. Soc., 137, 1000 (1990).

5. D. E. Rosner, Transport Processes in Chemically Reacting Flow Systems, Dover, New York (2000).

6. R. B. Bird, W. E. Steward, and E. N. Lightfoot, Transport Phenomena, John Wiley \& Sons, Hoboken, NJ (2002).

7. P. D. Neufeld, A. R. Janzen, and R. A. Aziz, J. Chem. Phys., 57, 1100 (1972).

8. W. L. Holstein, J. Electrochem. Soc., 135, 1788 (1988).

9. H. S. Heaton, W. C. Reynolds, and W. M. Kays, Int. J. Heat Mass Transfer, 7, 763 (1964).

10. B. Ceccaroli, Handbook of Photovoltaic Science and Engineering, pp. 153-205, John Wiley \& Sons, Hoboken, NJ (2003).

11. C. S. Herrick and D. W. Woodruff, J. Electrochem. Soc., 131, 2419 (1984).

12. H. Habuka, T. Nagoya, M. Mayusumi, M. Katayama, M. Shimada, and K Okuyama, J. Cryst. Growth, 169, 61 (1996).

13. F. Faller and A. Hurrle, IEEE Trans. Electron Devices, 46, 2048 (1999).

14. G. del Coso, I. Tobías, C. Cañizo, and A. Luque, J. Cryst. Growth, 299, 165 (2007).

15. T. Rummel, H.-F. Quast, and J. Haus (Siemens AG), U.S. Pat. 3232792 (1966) 